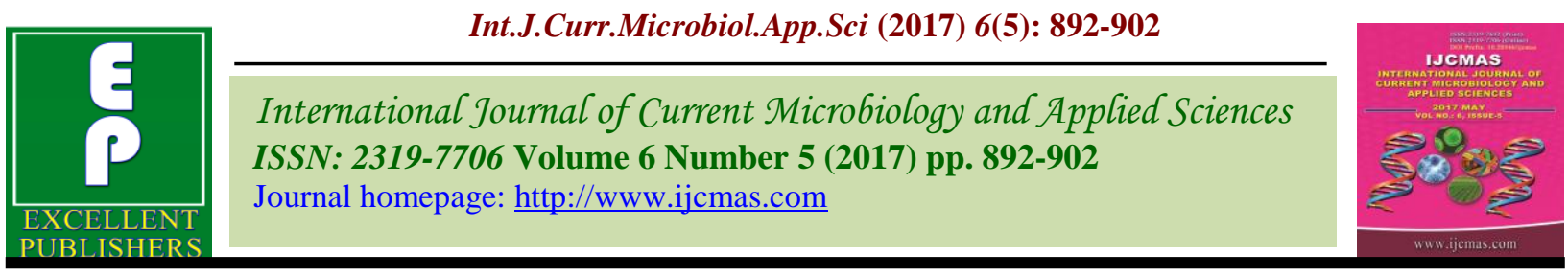

Original Research Article

https://doi.org/10.20546/ijcmas.2017.605.099

\title{
Influence of Storage Duration and Storage Temperature on In-Vitro Pollen Germination of Citrus Species
}

\author{
Shahnawaz Ahmed ${ }^{1}$, H.S. Rattanpal ${ }^{1}$, Eajaz Ahmad ${ }^{3}$ and Gurteg Singh ${ }^{1}$ \\ ${ }^{1}$ Division of Fruit Science, PAU, Ludhiana, Punjab - 141004, India \\ ${ }^{3}$ Department of Agronomy, Ludhiana, Punjab - 141004, India \\ *Corresponding author
}

\begin{abstract}
A B S T R A C T
Keywords

Citrus, In- vitro,

Pollen, Storage,

Temperature,

Species and

Germination.

Article Info

Accepted:

04 April 2017

Available Online:

10 May 2017

The aim of present study was to access the pollen in- vitro germination of pollen grains of five citrus species belonging to the family Rutaceae viz., mandarin (citrus. reticulata Blanco) sweet orange (C. sinensis L. Osbeck) grapefruit ( $C$. paradisi Macf.) pummelo ( $C$. maxima Burm. Merr.) and tangelo ( $C$. reticulata $\times C$. maxima or $\times C$. paradise) under low temperature storage conditions during year 2014. Pollen germination were checked up to 48 weeks, stored at different temperatures like $4^{\circ} \mathrm{C},-20^{\circ} \mathrm{C}$, and $-196^{\circ} \mathrm{C}$. Pollen stored at low temperature $\left(-196^{\circ} \mathrm{C}\right.$ and $\left.-20^{\circ} \mathrm{C}\right)$ showed better germination percentage as compared to pollen stored at $4^{0} \mathrm{C}$ and room temperature. The cultivar Mosambi had maximum average germination percentage when pollens were stored at $-196^{\circ} \mathrm{C}(70.8 \%)-,20^{\circ} \mathrm{C}(66.3 \%$, which was significantly higher than all other genotypes and followed by W.Murcott $196^{\circ} \mathrm{C}(60.9 \%)-20^{\circ} \mathrm{C}(48.5 \%)$. Minimum germination percentage was found in Marsh Seedless stored at $-196^{\circ} \mathrm{C}(21.7 \%)-20^{\circ} \mathrm{C}(12.5 \%)$. The results indicate that pollen collected and stored at sub-zero temperatures from early blooming citrus varieties can be stored for very long period without any appreciable loss of germination. The stored pollen can be used along the whole blooming season for hybridization programmes by fruit breeders.
\end{abstract}

\section{Introduction}

Citrus is one of the world's important fruit crop which is grown between latitude $35^{\circ} \mathrm{N} \sim 35^{\circ} \mathrm{S}$. Citrus comprises around 60 species, most of which are cultivated throughout the tropics and subtropics. They are indigenous in some parts of India, China, Northern Australia and New Caledonia (Harley et al., 2006). Citrus in India is grown in 1.07 million ha area with a total production of 11.14 million tonnes. The most important commercial citrus groups or cultivars in India are the mandarin (Citrus reticulata Blanco) sharing $41 \%$ followed by sweet orange (Citrus sinensis Osbeck) 23\% and acid lime
(Citrus aurintifolia Swingle), 23\% respectively (NHB, 2014).

One of the routine tasks in plant evolutionary biology, pollination biology and crop breeding research is determining total pollen grains per flower and proportion of viable pollen (Kevan, \& Husband, 2005 and Fang, Turner, Yan, Li, \& Siddique, 2010). The development of reliable methods for determining the functional quality of pollen helps in monitoring pollen vigour during storage, genetics and pollen-stigma interaction studies, crop improvement and 
breeding, and incompatibility and fertility studies (Dafni, 1992). The quality of pollen is assessed on the basis of viability and vigour of the pollen grain. Pollen vigour refers to the speed of germination of pollen grains and the rate of pollen tube growth (Ottaviano and Mulcahy, 1989).

In-vitro pollen germination tests have been used to determine the germination percentage of pollen and can also be used for assessing pollen vigour by monitoring the rate of germination over a period of time or the length of pollen tubes (Shivanna and Mohan Ram, 1993). Pollen which could not germinate usually shows poor tube growth which is likely to be ineffective in causing fertilization. Several methods of pollen storage have been tried of which the most important factors are controlled temperature and humidity Zhang (2002), Bomben et al., (2006), Song \& Tachibana (2007) and Dutta et al., (2013). Different investigators have same consensus that low temperature and humidity are the two major influencing factors in storage of pollen grains for a long period of time (Mesejo et al., 2006; Janick et al., 2010). Pollen grains can germinate and grow readily in water or in sugar medium, their growth can be accelerated by adding vitamins and microelements such as boron, magnesium, calcium, potassium etc. Pollen physiology especially germination and viability has received considerable attention for its application in plant breeding, conservation, adaptation and understanding of the physiological behaviour of the fertilizing pollen grains. There are several reports on pollen germination and viability of different taxa (Dafni \& Firmage, 2000; Vaknin et al., 2003; Ateyyeh, 2005; Bermejo et al., 2011 and Khan et al., 2013) with varied aims and objectives. Nair (1964) correlated pollen morphology of Vitis vinifera with physiological potential by studying pollen germination in different types. Mesejo et al.,
(2006) found out the inhibitory effect of $\mathrm{CuSO}_{4}$ on In vitro pollen germination of "Manderin" and after 8 hrs of incubation the development of pollen tube was stopped.

The In vitro pollen germination of Citrus maxima and $C$. paradisi was suppressed by adding olive oil to a medium containing $0.8 \%$ agar, $10 \%$ sucrose and 50 ppm Citric acid, but the germination was significantly increased in C. maxima in a medium having $0.8 \%$ agar and $20 \%$ sucrose (Ateyyah, 2005). Pollen viability and germination of these five Citrus species was assessed to develop a pollen bank from where desired pollen may be available for hybridization programmes. Present study was planned to access the in vitro pollen germination under different low temperature storage conditions.

\section{Materials and Methods}

Pollen from five citrus species viz. mandarin (citrus. reticulata Blanco) (Daisy, W.Murcott and Kinnow) sweet orange (C. sinensis $\mathrm{L}$. Osbeck) (Jaffa, Mosambi and Itaboria) grapefruit (C. paradisi Macf.) (Foster, Star Ruby and Marsh Seedless) pummelo ( $C$. maxima Burm. Merr.) (White Pummelo, Pink Pummelo and Devanpalli) and tangelo $(C$. reticulata $\times C$. maxima or $\times C$. paradise) (Minneola and Pearl) were collected from old orchard Punjab Agricultural University Ludhiana, Punjab for the use of this study. Flowers from male parents were collected at pre blooming stage (balloon stage) and were allowed to shed the pollen in shade for 3-4 hours under 100 watt lamps. Immediately after anther dehiscence, pollen were collected in vials and subjected to different storage conditions viz. room temperature (in anhydrous calcium chloride), $4^{\circ} \mathrm{C},-20^{\circ} \mathrm{C}$ and cryogenic storage in liquid nitrogen $\left(-196^{\circ} \mathrm{C}\right)$.

For in Vitro germination, pollen grains were germinated in 15 percent sucrose solution at 
weekly intervals. For this 1-2 drops of sucrose solution were placed in the cavity of a cavity slide and pollen grains were dusted over it by camel brush. The slides were covered with cover slip and edges were smeared by molten wax and slide was inverted instantly so as to form a hanging drop on the cover slip. The cavity slides were then placed in petridishes containing moist filter paper to ensure uniform and high relative humidity. Pollen tube growth was assessed for each genotype under microscope after $24 \mathrm{hrs}$ of incubation at $22 \pm 2^{\circ} \mathrm{C}$. The pollen grains having pollen tube at least two times longer than pollen diameter were considered to be germinated. Pollen germination was observed under digital microscope. Data were collected on four Petri dishes with at least 200 pollen grains in each one.

The experiment was carried out as completely randomized design (CRD). Data was analyzed using SAS software (SAS 9.3) and compression of means was carried out with Duncan's multiple range tests, considering $\mathrm{p} \leq 0.05$ as the level of significance.

\section{Results and Discussion}

\section{Pollen Germination}

Sweet orange (C. sinensis L. Osbeck) species showed $49.2 \%$ to $80.2 \%$ (Table:-1 and Fig:-1) germination of fresh pollen with maximum in Mosambi $(80.2 \%)$ followed by Jaffa $(65.1 \%)$ and minimum in Itaboria with $(49.2 \%)$. After 48 weeks of storage results obtained revealed that maximum average germination from $4^{\text {th }}$ to $48^{\text {th }}$ was noted as was noted as $70.8 \%$ in mosambi (which was significantly higher than all other genotypes $p \leq 0.05$ ) at freeze temperature $\left(-196^{\circ} \mathrm{C}\right)$ and minimum as $28.1 \%$ in Itaboria at refrigerated temperature $\left(4^{0} \mathrm{C}\right)$. The duration starts form 4 weeks to 48 weeks i.e. for whole one year and at 4 weeks the germination was found maximum in Mosambi $80.5 \%$ at freeze temperature $\left(-196^{\circ} \mathrm{C}\right)$ and after 48 weeks of storage the germination was found $58.2 \%$ where as Itaboria at 4 weeks shows lowest germination of $40.5 \%$ at refrigerated temperature $\left(4^{0} \mathrm{C}\right)$ and after 48 weeks germination was found $17.5 \%$ which is lowest among all sweet orange groups.

Mandarin (C. reticulate Blanco) species showed $69.7 \%$ to $75.5 \%$ (Table:-2 and Fig:-2) germination of fresh pollen with maximum in kinnow (75.5\%) followed by Murcott (72.6\%) and minimum in Daisy with (69.7\%). After 48 weeks of storage results obtained revealed that maximum average germination from $4^{\text {th }}$ to $48^{\text {th }}$ was noted as $61.2 \%$ in kinnow (which was significantly higher than all other genotypes $p \leq 0.05$ ) at freeze temperature ($196^{\circ} \mathrm{C}$ ) and minimum as $44.7 \%$ in Daisy at refrigerated temperature $\left(4^{0} \mathrm{C}\right)$. The duration starts form 4 weeks to 48 weeks i.e. for whole one year and at 4 weeks the germination was found maximum in Kinnow $75.3 \%$ at freeze temperature $\left(-196^{\circ} \mathrm{C}\right)$ and after 48 weeks of storage the germination was found $52.3 \%$ where as daisy at 4 weeks shows lowest germination of $60.5 \%$ at refrigerated temperature $\left(4^{0} \mathrm{C}\right)$ and after 48 weeks germination was found $28.5 \%$ which is lowest among all mandarin groups.

Grape fruit ( $C$. paradisi Macf.) species showed $30.5 \%$ to $42.6 \%$ (Table:-3 and Fig:-3) germination of fresh pollen with maximum in Star Ruby (42.6\%) followed by Foster $(38.5 \%)$ and minimum in Marsh Seedless with $(30.5 \%)$. After 48 weeks of storage results obtained revealed that maximum average germination from $4^{\text {th }}$ to $48^{\text {th }}$ was noted as $33.3 \%$ in Star Ruby (which was significantly higher than all other genotypes $p \leq 0.05$ ) at freeze temperature ($196^{\circ} \mathrm{C}$ ) and minimum as $12.5 \%$ in Marsh Seedless at refrigerated temperature $\left(4^{0} \mathrm{C}\right)$. The duration starts form 4 weeks to 48 Marsh Seedless weeks i.e. for whole one year and at 4 weeks the germination was found maximum 
in Star Ruby (42.0\%) at freeze temperature ($196^{\circ} \mathrm{C}$ ) and after 48 weeks of storage the germination was found $23.0 \%$ where as Marsh Seedless at 4 weeks shows lowest germination of $20.0 \%$ at refrigerated temperature $\left(4^{0} \mathrm{C}\right)$ and after 48 weeks germination was found $5.0 \%$ which is lowest among all grapefruit groups.

Pummelo (C. maxima Burm. Merr.) species showed $60.8 \%$ to $45.7 \%$ (Table:-4 and Fig:-4) germination of fresh pollen with maximum in Devanpalli (60.8\%) followed by White Pummelo (49.4\%) and minimum in Pink Pummelo with $(45.7 \%)$. After 48 weeks of storage results obtained revealed that maximum average germination from $4^{\text {th }}$ to $48^{\text {th }}$ was noted as $48.8 \%$ in devanpalli (which was significantly higher than all other genotypes $p \leq 0.05)$ at freeze temperature ($196^{\circ} \mathrm{C}$ ) and minimum as $23.4 \%$ in Pink Pummelo at refrigerated temperature $\left(4^{0} \mathrm{C}\right)$. The duration starts form 4 weeks to 48 Marsh Seedless weeks i.e. for whole one year and at 4 weeks the germination was found maximum in devanpalli $(60.0 \%)$ at freeze temperature ($196^{\circ} \mathrm{C}$ ) and after 48 weeks of storage the germination was found $40.0 \%$ where as Pink Pummelo at 4 weeks shows lowest germination of $36.4 \%$ at refrigerated temperature $\left(4^{0} \mathrm{C}\right)$ and after 48 weeks germination was found $12.4 \%$ which is lowest among all pummelo groups.

Tangelo $(C$. reticulata $\times C$. maxima or $\times C$. paradise) species showed $64.6 \%$ to $67.5 \%$ (Table:-5 and Fig:-5) germination of fresh pollen with maximum in Minneola $(67.5 \%)$ and minimum in Pearl with (64.6\%). After 48 weeks of storage results obtained revealed that maximum average germination from $4^{\text {th }}$ to $48^{\text {th }}$ was noted as $58.3 \%$ in Minneola (which was significantly higher than all other genotypes $p \leq 0.05)$ at freeze temperature ($196^{\circ} \mathrm{C}$ ) and minimum as $42.2 \%$ in Pearl at refrigerated temperature $\left(4^{0} \mathrm{C}\right)$. The duration starts form 4 weeks to 48 weeks i.e. for whole one year and at 4 weeks the germination was found maximum in Minneola $(67.4 \%)$ at freeze temperature $\left(-196^{\circ} \mathrm{C}\right)$ and after 48 weeks of storage the germination was found $49.5 \%$ where as Pearl at 4 weeks shows lowest germination of $57.0 \%$ at refrigerated temperature $\left(4^{0} \mathrm{C}\right)$ and after 48 weeks germination was found $24.5 \%$ which is lowest among all tangelo groups.

Pollen germination was examined up to 48 weeks in different storage conditions viz. 4 ${ }^{0} \mathrm{C},-20{ }^{0} \mathrm{C}$ and $-196{ }^{0} \mathrm{C}$ (Table and Figure). The results obtained revealed that pollen germination was more than 30.00 per cent in all the fourteen cultivars immediately after gathering in laboratory with maximum in Mosambi (80.2\%) (which was significantly higher than all other genotypes $p \leq 0.05$ ) followed by Kinnow (75.5\%) and minimum in Marsh Seedless $(30.5 \%)$. While comparing different storage temperatures, the viability was lost immediately after storage in case of room temperature. However, maximum viability was observed at sub zero storage temperatures $\left(-196^{\circ} \mathrm{C}\right)$ during the whole period of storage duration with highest average pollen viability was noted in cultivar Mosambi $(75.7 \%)$ (Table:-6) at freeze temperature $\left(-196^{\circ} \mathrm{C}\right)$ from $4^{\text {th }}$ to $48^{\text {th }}$ week (which was significantly higher than all other genotypes $p \leq 0.05$ ) and lowest in Marsh seedless (25.7\%) at refrigerated temperature $\left(4^{0} \mathrm{C}\right)$. Maximum pollen germination among all genotypes was found in Mosambi $(80.5 \%)$ at freeze temperature $\left(-196^{\circ} \mathrm{C}\right)$ at $4^{\text {th }}$ week of storage and minimum was found in Marsh Seedless $(5.0 \%)$ at refrigerated temperature $\left(4^{0} \mathrm{C}\right)$ on $48^{\text {th }}$ week of storage. Fresh pollens have more germination than the stored one. Eti (1991); Parfitt and Almedhi (1984); Seiheimer and Stover (1982) have indicated that germination percentage vary significantly according to fruit species or cultivars. 
Table.1 pollen germination \% of sweet orange (C. sinensis L. Osbeck) varieties

\begin{tabular}{|c|c|c|c|c|c|c|c|c|c|}
\hline & \multicolumn{3}{|c|}{ Mosambi } & \multicolumn{3}{|l|}{ Jaffa } & \multicolumn{3}{|c|}{ Itaboria } \\
\hline $\begin{array}{l}\text { Duration } \\
\text { (weeks) }\end{array}$ & $4^{\circ} \mathrm{C}$ & $-20^{\circ} \mathrm{C}$ & $196^{\circ} \mathrm{C}$ & $4^{\circ} \mathrm{C}$ & $-20^{\circ} \mathrm{C}$ & $-196^{\circ} \mathrm{C}$ & $4^{\circ} \mathrm{C}$ & $-20^{\circ} \mathrm{C}$ & $196^{\circ} \mathrm{C}$ \\
\hline W4 & 70.4 & 76.3 & 80.5 & 55.3 & 61.0 & 65.1 & 40.5 & 46.0 & 49.5 \\
\hline W8 & 64.4 & 72.0 & 77.3 & 50.2 & 60.0 & 64.4 & 35.4 & 45.0 & 47.4 \\
\hline W12 & 63.1 & 71.0 & 76.2 & 49.4 & 58.0 & 63.2 & 34.1 & 43.7 & 46.3 \\
\hline W16 & 62.4 & 70.0 & 75.1 & 48.3 & 57.0 & 61.2 & 32.4 & 42.0 & 44.4 \\
\hline W20 & 60.5 & 68.0 & 74.2 & 46.3 & 55.0 & 60.3 & 30.5 & 40.0 & 43.3 \\
\hline W24 & 58.5 & 67.0 & 73.3 & 45.5 & 54.0 & 58.4 & 29.2 & 39.0 & 41.5 \\
\hline W28 & 56.4 & 64.7 & 71.3 & 43.3 & 52.0 & 57.1 & 27.3 & 37.7 & 40.4 \\
\hline W32 & 53.4 & 64.0 & 69.2 & 42.4 & 49.0 & 55.1 & 25.3 & 36.0 & 39.4 \\
\hline W36 & 51.2 & 62.0 & 67.3 & 40.5 & 47.0 & 54.4 & 23.4 & 34.0 & 38.3 \\
\hline W40 & 50.1 & 63.0 & 64.3 & 39.1 & 45.0 & 51.4 & 21.2 & 31.7 & 37.1 \\
\hline W44 & 48.4 & 61.0 & 62.3 & 37.4 & 43.0 & 49.4 & 20.1 & 31.0 & 35.4 \\
\hline W48 & 46.4 & 56.0 & 58.2 & 35.5 & 40.0 & 47.1 & 17.5 & 28.0 & 32.6 \\
\hline $\begin{array}{l}\text { LSD } \\
(p \leq 0.05)\end{array}$ & $\begin{array}{l}\text { LSD (V } \\
\text { LSD (W } \\
\text { LSD (Te }\end{array}$ & $\begin{array}{l}\text { ety) }=1 \\
\text { k) }=2 . \\
\text { perature) }=\end{array}$ & & & $\begin{array}{l}\text { LSD (Variet } \\
\text { LSD (Variet } \\
\text { LSD (Week } \\
\text { LSD (Variet }\end{array}$ & $\begin{array}{l}\text { x week) } \\
\text { x Tempera } \\
\text { Temperatu } \\
\text { x week x T }\end{array}$ & $\begin{array}{l}\text { Ire) } \\
\text { e) } \\
\text { mperatu }\end{array}$ & $\begin{array}{l}=3.5(\mathrm{NS}) \\
=1.76(\mathrm{NS}) \\
=3.51(\mathrm{NS}) \\
=6.09(\mathrm{NS})\end{array}$ & \\
\hline
\end{tabular}

\begin{tabular}{|l|l|l|l|}
\hline Fresh pollen Germination (\%) at room temperature & 65.1 & 80.2 & 49.2 \\
\hline After one week pollen germination \% at room temperature & 00.00 & 00.00 & 00.00
\end{tabular}

Table.2 pollen germination \% of Mandrin (C. reticulate Blanco) varieties

\begin{tabular}{|c|c|c|c|c|c|c|c|c|c|}
\hline & \multicolumn{3}{|l|}{ Kinnow } & \multicolumn{3}{|l|}{ Daisy } & \multicolumn{3}{|c|}{ W. Murcoutt } \\
\hline $\begin{array}{l}\text { Duration } \\
\text { (weeks) }\end{array}$ & $4^{\circ} \mathrm{C}$ & $-20^{\circ} \mathrm{C}$ & $-196^{\circ} \mathrm{C}$ & $4^{\mathrm{o}} \mathrm{C}$ & $-20^{\circ} \mathrm{C}$ & $-196^{\circ} \mathrm{C}$ & $4^{\circ} \mathrm{C}$ & $-20^{\circ} \mathrm{C}$ & $196^{\circ} \mathrm{C}$ \\
\hline W4 & 62.4 & 68.0 & 75.3 & 60.5 & 64.3 & 69.0 & 63.3 & 65.3 & 72.0 \\
\hline W8 & 57.3 & 63.0 & 67.4 & 53.4 & 60.5 & 66.0 & 56.4 & 63.4 & 67.3 \\
\hline W12 & 56.4 & 62.0 & 66.3 & 52.3 & 59.4 & 65.0 & 55.3 & 62.3 & 66.0 \\
\hline W16 & 54.4 & 58.0 & 63.4 & 51.3 & 57.4 & 64.0 & 54.2 & 58.3 & 64.0 \\
\hline W20 & 53.5 & 55.0 & 62.5 & 49.5 & 54.4 & 63.0 & 52.3 & 55.5 & 63.0 \\
\hline W24 & 50.5 & 51.0 & 60.4 & 46.6 & 50.5 & 61.0 & 50.4 & 50.5 & 62.0 \\
\hline W28 & 47.4 & 47.0 & 59.4 & 43.2 & 46.5 & 60.0 & 48.6 & 47.3 & 60.0 \\
\hline W32 & 43.4 & 45.0 & 58.2 & 40.5 & 43.5 & 59.0 & 46.5 & 45.5 & 59.0 \\
\hline W36 & 40.5 & 43.0 & 57.4 & 38.5 & 41.5 & 57.0 & 42.3 & 43.5 & 57.0 \\
\hline W40 & 39.4 & 42.0 & 56.4 & 36.5 & 39.5 & 55.0 & 40.5 & 40.4 & 56.0 \\
\hline W44 & 36.4 & 40.0 & 55.1 & 35.3 & 36.5 & 54.0 & 39.4 & 39.5 & 55.0 \\
\hline W48 & 30.4 & 34.0 & 52.3 & 28.5 & 30.5 & 50.0 & 32.4 & 35.5 & 50.0 \\
\hline $\begin{array}{l}\text { LSD } \\
(p \leq 0.05)\end{array}$ & $\begin{array}{l}\text { LSD (Va } \\
\text { LSD (We } \\
\text { LSD (Te }\end{array}$ & $\begin{array}{l}\text { ty) }= \\
\text { erature) }\end{array}$ & $\begin{array}{l}1.18 \\
.35 \\
1.77\end{array}$ & & $\begin{array}{l}\text { LSD (Va } \\
\text { LSD (Va } \\
\text { LSD (We } \\
\text { LSD (Va }\end{array}$ & $\begin{array}{l}\text { ety } \mathrm{x} \text { weel } \\
\text { ety } \mathrm{x} \text { Tem } \\
\mathrm{k} \times \mathrm{Temp} \\
\text { ety } \mathrm{x} \text { weel }\end{array}$ & $\begin{array}{l}\quad=4.08( \\
\text { erature) }= \\
\text { rature) }=4 \\
x \text { Temper }\end{array}$ & $\begin{array}{l}\text { S) } \\
04(\mathrm{NS}) \\
8 \text { (NS) } \\
\text { are) }=7.06\end{array}$ & \\
\hline
\end{tabular}

\begin{tabular}{|l|l|l|l|}
\hline Fresh pollen Germination (\%) at room temperature & 75.5 & 69.7 & 72.6 \\
\hline After one week pollen germination \% at room temperature & 00.00 & 00.00 & 00.00 \\
\hline
\end{tabular}


Table.3 Pollen germination \% of Grapefruit (C. paradisi Macf.) varieties

\begin{tabular}{|c|c|c|c|c|c|c|c|c|c|}
\hline & \multicolumn{3}{|c|}{ Foster } & \multicolumn{3}{c|}{ Star Ruby } & \multicolumn{3}{c|}{ Marsh Seedless } \\
\hline $\begin{array}{c}\text { Duration } \\
\text { (weeks) }\end{array}$ & $4^{\circ} \mathrm{C}$ & $-20^{\circ} \mathrm{C}$ & $-196^{\circ} \mathrm{C}$ & $4^{\circ} \mathrm{C}$ & $-20^{\circ} \mathrm{C}$ & $-196^{\circ} \mathrm{C}$ & $4^{\circ} \mathrm{C}$ & $-20^{\circ} \mathrm{C}$ & $-196^{\circ} \mathrm{C}$ \\
\hline $\mathrm{W} 4$ & 30.2 & 35.0 & 38.6 & 31.7 & 38.0 & 42.0 & 20.0 & 26.5 & 30.5 \\
\hline $\mathrm{W} 8$ & 26.5 & 32.0 & 35.5 & 28.0 & 35.0 & 40.0 & 18.0 & 24.6 & 27.4 \\
\hline $\mathrm{W} 12$ & 25.4 & 31.0 & 34.3 & 27.0 & 34.0 & 39.0 & 17.0 & 22.5 & 26.2 \\
\hline $\mathrm{W} 16$ & 24.2 & 30.0 & 32.5 & 26.0 & 33.0 & 38.0 & 16.0 & 21.2 & 25.3 \\
\hline $\mathrm{W} 20$ & 23.1 & 29.0 & 31.3 & 23.0 & 32.0 & 37.0 & 15.0 & 19.5 & 23.6 \\
\hline $\mathrm{W} 24$ & 22.5 & 26.0 & 29.4 & 21.0 & 28.0 & 35.0 & 13.0 & 17.5 & 22.2 \\
\hline $\mathrm{W} 28$ & 21.1 & 24.0 & 28.1 & 20.3 & 26.0 & 32.0 & 12.3 & 16.2 & 21.3 \\
\hline $\mathrm{W} 32$ & 19.4 & 23.3 & 26.4 & 19.0 & 25.0 & 30.7 & 10.0 & 14.4 & 20.4 \\
\hline $\mathrm{W} 36$ & 17.2 & 21.0 & 25.4 & 17.0 & 23.7 & 30.0 & 9.0 & 13.2 & 19.2 \\
\hline $\mathrm{W} 40$ & 15.3 & 20.0 & 23.4 & 14.7 & 21.0 & 27.0 & 8.0 & 12.1 & 17.5 \\
\hline $\mathrm{W} 44$ & 13.4 & 18.0 & 22.1 & 13.7 & 20.0 & 26.0 & 7.0 & 11.3 & 15.4 \\
\hline $\mathrm{W} 48$ & 10.6 & 15.0 & 20.5 & 10.0 & 16.0 & 23.0 & 5.0 & 8.5 & 11.4 \\
\hline & \multicolumn{3}{|l|}{$\begin{array}{l}\text { LSD (Variety) }=1.24 \\
\text { LSD (Week) }\end{array}=2.48$} \\
$\begin{array}{l}\text { LSD (Temperature) }=1.24 \\
(\mathrm{p} \leq 0.05)\end{array}$
\end{tabular}

\begin{tabular}{|l|l|l|l|}
\hline Fresh pollen Germination (\%) at room temperature & 38.5 & 42.6 & 30.5 \\
\hline After one week pollen germination \% at room temperature & 00.00 & 00.00 & 00.00 \\
\hline
\end{tabular}

Table.4 Pollen germination \% of Pummelo (C. maxima Burm. Merr.) varieties

\begin{tabular}{|c|c|c|c|c|c|c|c|c|c|}
\hline & \multicolumn{3}{|c|}{ Pink Pumello } & \multicolumn{3}{|c|}{ White Pumello } & \multicolumn{3}{|c|}{ Devanpalli } \\
\hline $\begin{array}{c}\text { Duration } \\
\text { (weeks) }\end{array}$ & $4^{\circ} \mathrm{C}$ & $-20^{\circ} \mathrm{C}$ & $-196^{\circ} \mathrm{C}$ & $4^{\circ} \mathrm{C}$ & $-20^{\circ} \mathrm{C}$ & $-196^{\circ} \mathrm{C}$ & $4^{\circ} \mathrm{C}$ & $-20^{\circ} \mathrm{C}$ & $-196^{\circ} \mathrm{C}$ \\
\hline W4 & 36.4 & 42.3 & 45.0 & 40.4 & 46.5 & 49.0 & 50.4 & 55.3 & 60.0 \\
\hline W8 & 30.5 & 40.3 & 43.0 & 32.5 & 42.4 & 46.0 & 43.4 & 52.4 & 55.0 \\
\hline W12 & 29.2 & 39.4 & 42.0 & 31.5 & 41.2 & 45.0 & 41.4 & 51.2 & 54.0 \\
\hline W16 & 28.5 & 38.4 & 41.0 & 29.2 & 40.5 & 43.0 & 40.4 & 50.3 & 53.0 \\
\hline W20 & 26.5 & 36.3 & 40.0 & 28.2 & 39.4 & 42.0 & 39.1 & 49.2 & 50.0 \\
\hline W24 & 23.5 & 34.1 & 38.0 & 26.5 & 37.1 & 40.0 & 38.3 & 47.5 & 49.0 \\
\hline W28 & 21.4 & 33.5 & 37.0 & 25.3 & 36.5 & 39.0 & 35.3 & 45.5 & 48.0 \\
\hline W32 & 20.2 & 31.4 & 35.0 & 24.2 & 35.1 & 38.0 & 33.5 & 43.6 & 46.0 \\
\hline W36 & 19.1 & 30.3 & 33.0 & 23.3 & 32.4 & 36.0 & 31.3 & 42.4 & 45.0 \\
\hline W40 & 17.4 & 29.5 & 32.0 & 21.6 & 30.6 & 35.0 & 30.5 & 40.5 & 44.0 \\
\hline W44 & 15.4 & 27.4 & 29.7 & 20.5 & 29.2 & 33.0 & 28.5 & 39.5 & 42.0 \\
\hline W48 & 12.4 & 25.4 & 27.0 & 17.5 & 26.5 & 30.0 & 25.4 & 35.5 & 40.0 \\
\hline $\begin{array}{l}\text { LSD } \\
(p \leq 0.05)\end{array}$ & \multicolumn{4}{|c|}{$\begin{array}{l}\text { LSD }(\text { Variety })=1.16 \\
\text { LSD }(\text { Week })=2.31 \\
\operatorname{LSD}(\text { Temperature })=1.16\end{array}$} & \multicolumn{5}{|c|}{$\begin{array}{l}\text { LSD }(\text { Variety } x \text { week }) \quad=4.00(\mathrm{NS}) \\
\text { LSD }(\text { Variety } \times \text { Temperature })=2.00(\mathrm{NS}) \\
\text { LSD }(\text { Week } \times \text { Temperature })=4.00 \\
\text { LSD }(\text { Variety } \times \text { week } \times \text { Temperature })=6.93\end{array}$} \\
\hline
\end{tabular}

\begin{tabular}{|l|l|l|l|}
\hline Fresh pollen Germination (\%) at room temperature & 49.4 & 45.7 & 60.8 \\
\hline After one week pollen germination \% at room temperature & 00.00 & 00.00 & 00.00 \\
\hline
\end{tabular}


Table.5 Pollen germination $\%$ of Tangelo (C. reticulata $\times C$. maxima or $\times C$. paradise $)$ varieties

\begin{tabular}{|c|c|c|c|c|c|c|}
\hline & \multicolumn{3}{|c|}{ Minneola } & \multicolumn{3}{|c|}{ Pearl } \\
\hline $\begin{array}{c}\text { Duration } \\
\text { (weeks) }\end{array}$ & $4^{\circ} \mathrm{C}$ & $-20^{\circ} \mathrm{C}$ & $-196^{\circ} \mathrm{C}$ & $4^{\circ} \mathrm{C}$ & $-20^{\circ} \mathrm{C}$ & $-196^{\circ} \mathrm{C}$ \\
\hline W4 & 58.6 & 62.0 & 67.4 & 57.0 & 60.0 & 64.0 \\
\hline W8 & 54.4 & 60.0 & 64.5 & 51.0 & 58.0 & 61.0 \\
\hline W12 & 53.4 & 59.0 & 63.4 & 50.0 & 57.0 & 60.0 \\
\hline W16 & 52.3 & 57.0 & 62.2 & 49.0 & 55.0 & 59.0 \\
\hline W20 & 50.4 & 53.0 & 60.5 & 48.0 & 53.0 & 58.0 \\
\hline W24 & 46.5 & 49.0 & 59.4 & 45.0 & 51.0 & 55.0 \\
\hline W28 & 40.3 & 47.0 & 57.3 & 42.0 & 49.0 & 53.0 \\
\hline W32 & 38.5 & 45.0 & 55.5 & 39.0 & 47.0 & 52.0 \\
\hline W36 & 36.3 & 43.0 & 54.3 & 36.0 & 46.0 & 50.0 \\
\hline W40 & 33.4 & 40.0 & 53.3 & 33.0 & 42.0 & 49.0 \\
\hline W44 & 30.3 & 38.0 & 52.2 & 30.0 & 39.0 & 47.0 \\
\hline W48 & 24.5 & 31.0 & 49.5 & 26.0 & 35.0 & 42.0 \\
\hline $\operatorname{LSD}(\mathrm{p} \leq 0.05)$ & \multicolumn{3}{|c|}{$\begin{array}{ll}\text { LSD }(\text { Variety) } & =1.14 \\
\text { LSD }(\text { Week }) & =2.79 \\
\text { LSD }(\text { Temperature })=1.39\end{array}$} & \multicolumn{3}{|c|}{$\begin{array}{ll}\text { LSD }(\text { Variety x week) } & =3.94(\mathrm{NS}) \\
\text { LSD }(\text { Variety x Temperature }) & =1.97 \\
\text { LSD }(\text { Week x Temperature }) & =4.83 \\
\text { LSD }(\text { Variety x week x Temperature })=6.83(\mathrm{NS})\end{array}$} \\
\hline
\end{tabular}

\begin{tabular}{|l|l|l|}
\hline Fresh pollen Germination (\%) at room temperature & 67.5 & 64.6 \\
\hline After one week pollen germination \% at room temperature & 00.00 & 00.00 \\
\hline
\end{tabular}

Table.6 Combined interaction of all varieties, weeks and temperature among each other and their values

\begin{tabular}{|c|c|c|}
\hline $\begin{array}{l}\text { LSD } \\
(\mathrm{p} \leq 0.05)\end{array}$ & $\begin{array}{l}\text { LSD }(\text { Variety) }=1.15 \\
\text { LSD }(\text { Week })=1.06 \\
\text { LSD }(\text { Temperature })=0.53\end{array}$ & $\begin{array}{ll}\text { LSD }(\text { Variety x week) } & =3.96(\mathrm{NS}) \\
\text { LSD }(\text { Variety x Temperature) } & =1.98 \\
\text { LSD (Week x Temperature) } & =1.83 \\
\text { LSD (Variety x week x Temperature) }=6.85\end{array}$ \\
\hline
\end{tabular}

Fig.1 Pollen germination \% of sweet orange (C. sinensis L. Osbeck) varieties

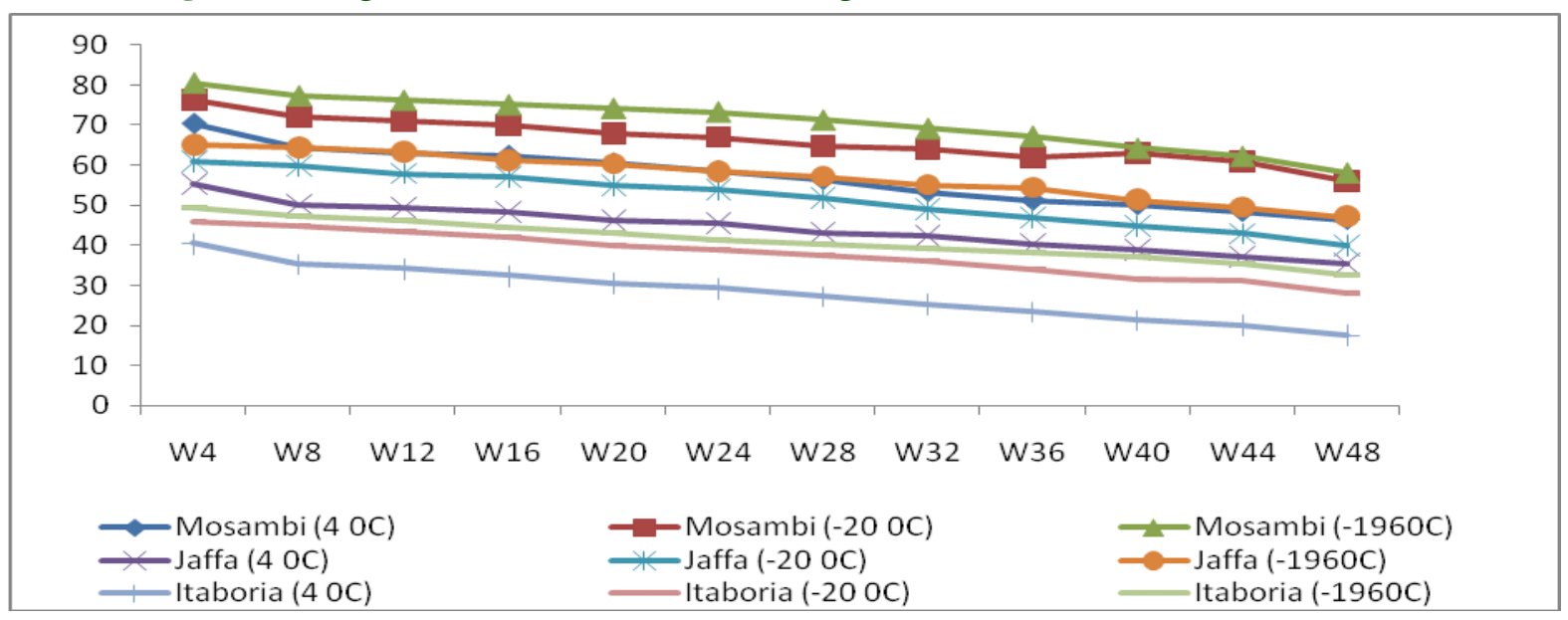


Fig. 2 pollen germination \% of Mandrin (C. reticulate Blanco) varieties

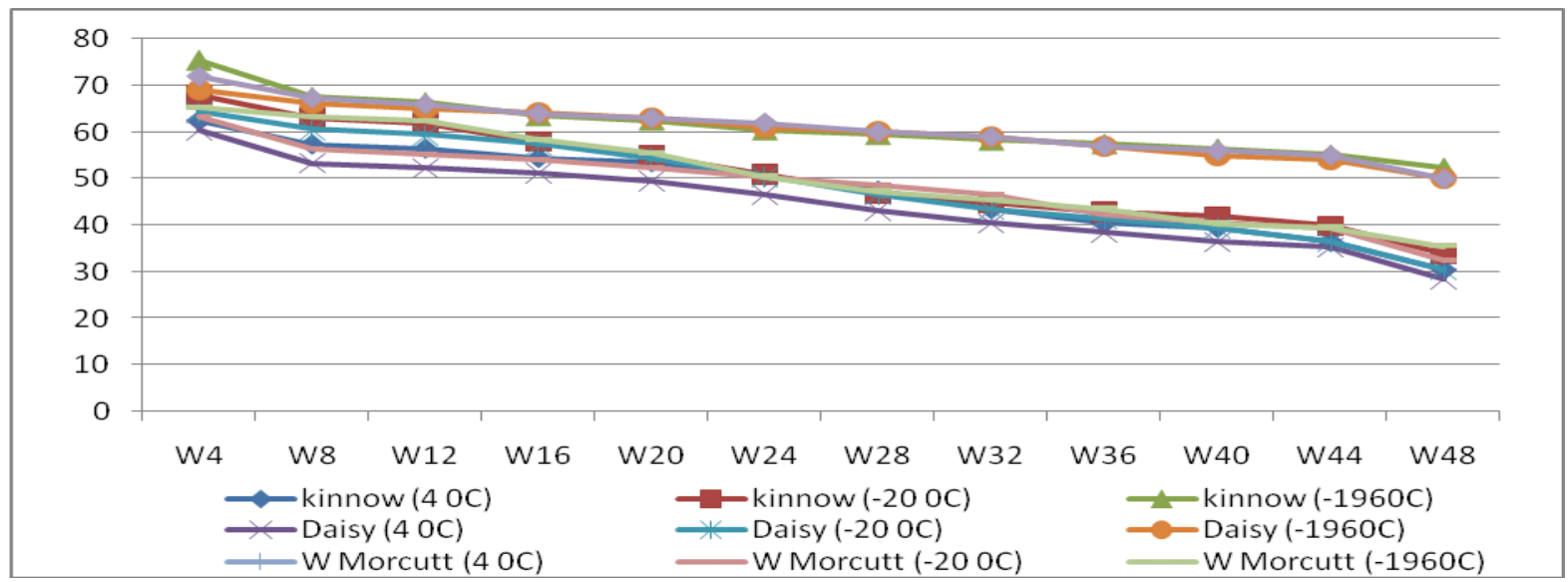

Fig.3 pollen germination \% of Grapefruit (C. paradisi Macf.) varieties

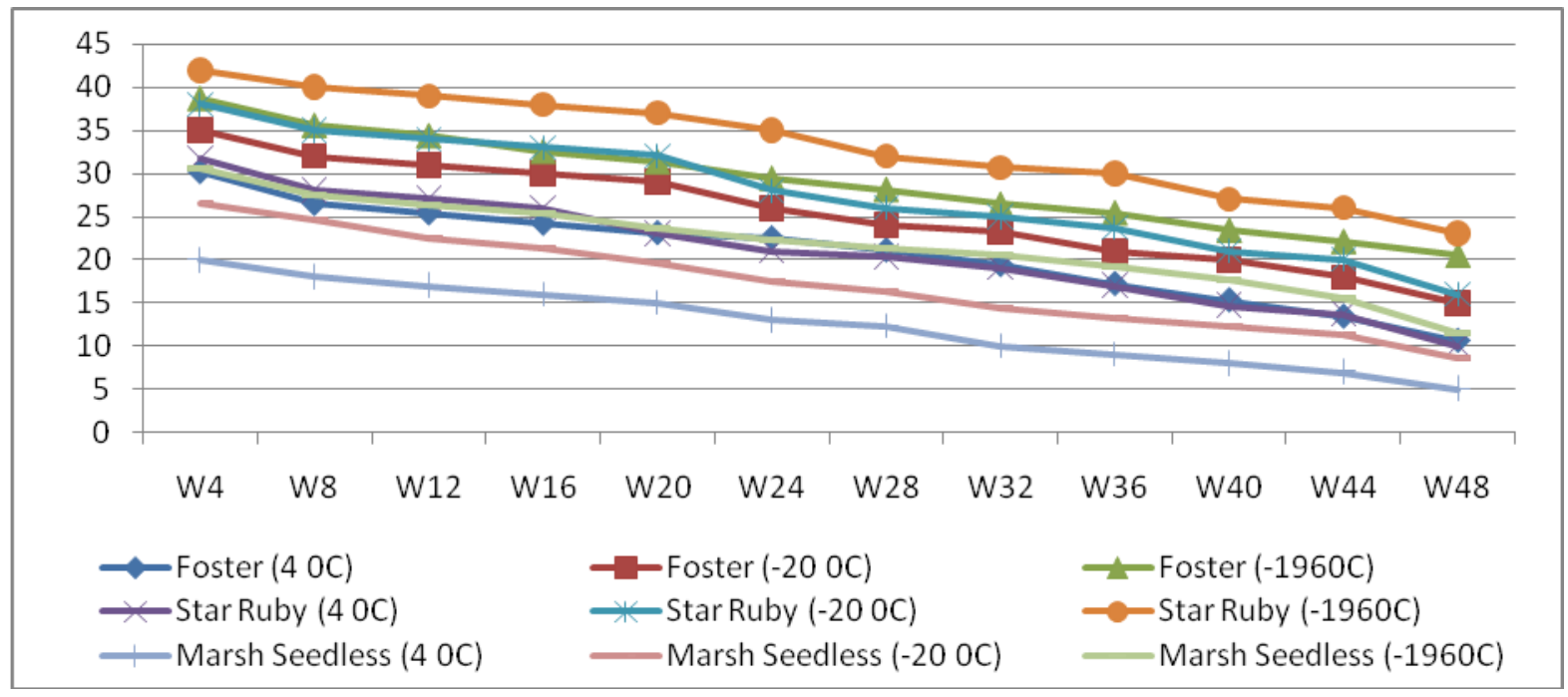

Fig.4 pollen germination \% of Pummelo (C. maxima Burm. Merr.) varieties

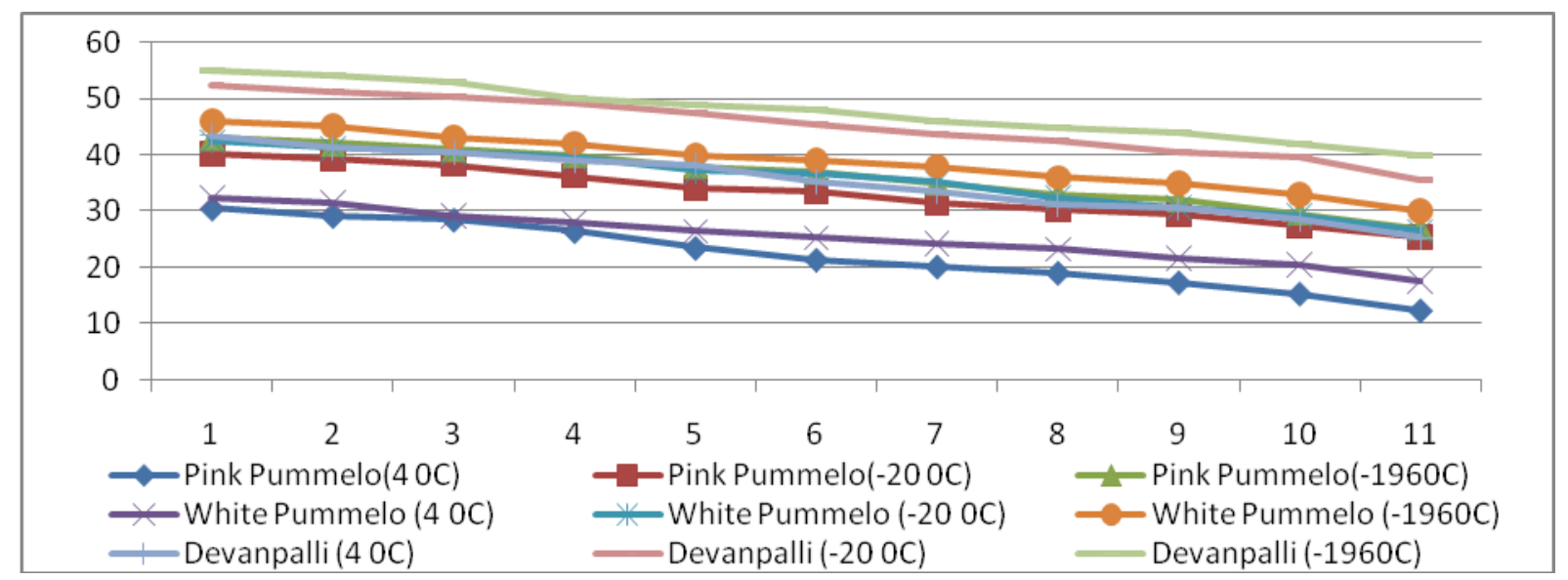


Fig.5 pollen germination \% of Tangelo (C. reticulata $\times$ C. maxima or $\times$ C. paradise $)$ varieties

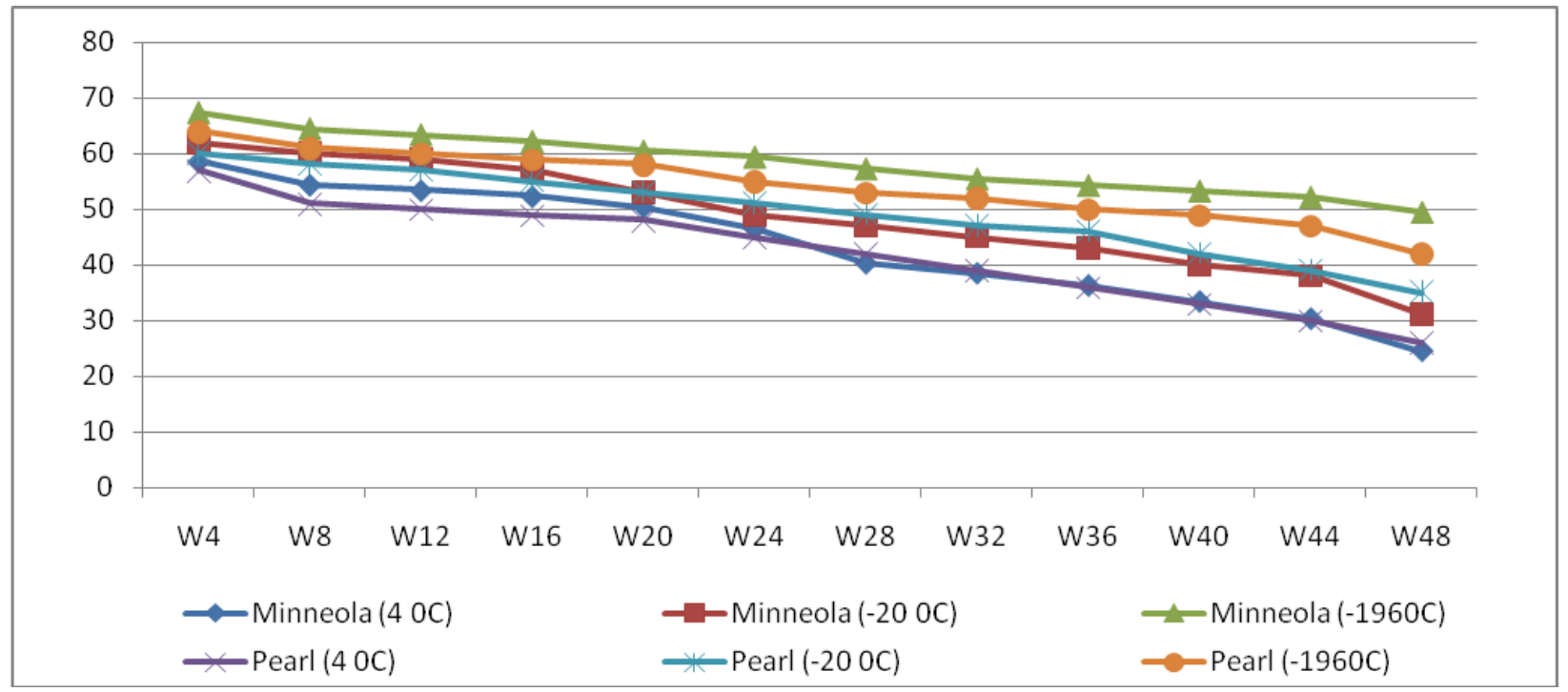

Higher values for pollen germination at low storage temperature and decline at high storage temperature were observed by several workers (Hanna and Towill, 1995; Perveen et al., 2007; Perveen and Khan, 2008). It is quite obvious that staining tests are not reliable measure of pollen viability as compared to in vitro pollen germination tests. Hence a combination of both viability and germination tests will provide a better understanding about the pollen behaviour. In the present study wide variations in viability and germination of pollen grains was observed with different storage temperatures and durations among different citrus cultivars. This variability may be due to pollen fertility, as a result of regular meiosis and activation of certain enzyme systems present in the pollen grain itself. Besides genotype and environmental interactions may also play an important role. This phenomenon indicates genetic differences among the genotypes which have been reported by many researchers in many of the fruit tree species and cultivars (Alburquerque et al., 2007; Sharafi et al., 2011). Anjum and Shaukat (2014) five Citrus species while studying pollen germination beyond 48 weeks in the refrigerator $\left(4^{\circ} \mathrm{C}\right)$, freezer $\left(-20^{\circ} \mathrm{C},-30^{\circ} \mathrm{C}\right)$ and freeze drier $\left(-60^{\circ} \mathrm{C}\right)$ the best method to maintain pollen seems to be freeze drier ($60^{\circ} \mathrm{C}$ ) and the viability of stored pollen grains for a long period of time. Among five species Citrus aurantium, C. limon and C. sinensis showed high percentage of germination as compared to $C$. reticulata and $C$. paradisii. The gradual loss of germination at low temperatures $\left(-20^{\circ} \mathrm{C}\right.$ and $\left.-196^{\circ} \mathrm{C}\right)$ observed in the present study may be attributed to frequent freezing and thawing of pollen grains. Gubley et al., 2015 concluded that the pollen of pollen of 'Lamas' and 'Meyer' varieties of lemon had the highest in vitro germination with $39.77 \%$ and $39.04 \%$, respectively. Furthermore low temperature might have lead to intracellular ice formation, cell death and thereby loss of germination. Our results clearly indicated that it is feasible to store pollen grains of citrus at sub zero temperatures without any significant loss in their viability and germinability, and they may be used effectively throughout the flowering season for assisted pollination so as to broaden the citrus genetic base.

\section{Acknowledgements}

This work was by financial supported 
scholarships fellowship (DST INSPIRE "Innovation in Science Pursuit for Inspired Research"), Ministry of Science and Technology, Department of Science and Technology Technology Bhawan, New Mehrauli road New Delhi-110016

\section{References}

Alburquerque, N., García Montiel, F., Burgos, L. 2007. Influence of storage temperature on the viability of sweet cherry pollen. Spanish J. Agric. Res., 5: 86-90.

Ateyyeh, A.F. 2005. Improving In vitro pollen germination of five species of fruit trees. Agri. Sci., 33(2): 189-194.

Bermejo, Almudena, J., Pardo, Cano and Antonio. 2011. Infulence of gamma irradiation on seedless Citrus production: pollen germination and fruit quality. Food and Nutri. Sci., 2(3): 169180.

Bomben, C., C. Malossini, G. Cipriani and R. Testolin. 2006. Long term storage of Kiwifruit pollen. Acta Hort., 498: IV International Symposium on Kiwifruit.

Dafni, A. 1992. Pollination Ecology: A practical approach. New York, New York: Oxford University Press.

Dafni, A. and D. Firmage. 2000. Pollen viability and longevity: practical, ecological and evolutionary amplications. Pl. Syst. Evol., 222: 113132.

Dafni, A., Kevan, P.G., \& Husband, B.C. 2005. Practical Pollination Biology. Enviroquest Ltd., Ontario, Canada.

Dutta, S.K., M. Srivatav, R. Chaudhary, K. Lal, P. Patil. S.K. Sing and A.K. Sing. 2013. Low temperature storage of mango. Mangifera indica L.), pollen. Sci. Hort., 161: 193-197.

Eti, S. 1991. Determination of pollen viability and germination capability of some fruit species and varieties in vitro conditions.
J. Agric. Fac. Cukorova Univ, 6: 69-80. Fang, X., Turner, N.C., Yan, G., Li, F., \& Siddique, K.H.M. 2010. Flower numbers, pod production, pollen viability, and pistil function are reduced and flower and pod abortion increased in chickpea. Cicerarietinum L.) under terminal drought. J. Experimental Bot., 61 , 335-345. http://dx.doi.org/10.1093/jxb/erp307.

Gulay Demir, Ertugrul Turgutoglu and Senay Kurt. 2015. Assessment of pollen viability and germination in seven varieties of lemon Ekin, J. Crop Breeding and Genetics, 1(1): 47-49.

Hanna, W.W. and L.E. Towil. 1995. Longterm pollen storage. Plant Breeding Rev., 13: 179-207.

Harley, I., Manner, Richard, S., Buker, Virginia Easton Smith, Deborah Ward, and Craig R. Elevitch. 2006. Species Profiles for Pacific Island Agroforestry. www.traditionaltree.org) 1-35.

Janick, J.W.W. Hanna and L.E. Towill. 2010. Long term pollen storage. Plant Breeding Rev., 13.

Khan, S.A., A. Perveen and G.R. Sarwar. 2013. Germination capacity and viability in pollen of Prunus amygdalus Batsch. Rosaceae. Pak. J. Bot., 45(4): 1383-1385.

Mesejo, C., A.M. Fuentes, C. Reig and R. Fernando. 2006. The inhibitory effect of $\mathrm{CuSo} 4$ on Citrus pollen germination and pollen tube growth and its application for the production of seedless fruit.

Nair, P.K.K. 1964. Pollen grains of Western Himalayan plants, Asia Publishing House Bombay.

NHB. 2014. NHB Database for fruit crops. Gurgaon, India.

Ottaviano, E., and D.L. Mulcahy, 1989. Genetics of angiosperm pollen. $A d v$. Genet., 26: 1-64.

Parfitt, D.E. and Almehdi, A. 1984. Liquid 
nitrogen storage of pollen from five cultivated Prunus species. Hort. Sci., 19: 69-70.

Perveen, A. and S.A. Khan. 2008. Germination capacity of stored pollen of Malus pumila L. Rosaceae) and their maintenance. Pak. J. Bot., 40(3): $963-$ 966.

Perveen, A., S.A. Khan and R. Abid. 2007. Maintenance of pollen germination capacity of carica papaya L. Caricaceae. Pak. J. Bot., 39(6): 1403-1406.

Seilheimer, M. and Stösser, R. 1982. Die Eignung verschiedener Apfelsorten als Pollenspender. Erwerbsobstbau, 24: 62-65.

Shaukat Ali Khan and Anjum Parveen. 2014. in vitro pollen germination of five citrus species, Pak. J. Bot., 46(3): 951-956.

Shivanna, K.R. and Mohan Ram, H.Y. 1993.
Pollination biology: contributions to fundamental and applied aspects, Curr. Sci., 65(3): 226-233.

Song, J. and S. Tachibana. 2007. Loss of viability of tomato pollen during long term dry storage is associated with reduced capacity for translating polyamine biosynthetic enzyme genes after rehydration. J. Exp. Bot., 58(1516): 4235-4244.

Vaknin, Y., D. Mills and A. Benzioni. 2003. Pollen production and pollen viability in male jojoba plants. Industrial Crops and Products, 18(2): 117-123.

Yavar Sharafi, Ali-Reza Motallebbi-Azar and Ali Bahmani. 2011. African $J$. Biotechnol., 10(41): 8064-8069.

Zhang, L.Y. 2002. Conervation of pollen at low temperature and in liquid Nitrogen. Acta. Hort. Sinica, 2000-02.

\section{How to cite this article:}

Shahnawaz Ahmed, H.S. Rattanpal, Eajaz Ahmad and Gurteg Singh. 2017. Influence of Storage Duration and Storage Temperature on In- Vitro Pollen Germination of Citrus Species. Int.J.Curr.Microbiol.App.Sci. 6(5): 892-902. doi: https://doi.org/10.20546/ijcmas.2017.605.099 